\title{
CONTORNO DE LÍNGUA DE CRIANÇAS BRASILEIRAS COM DESVIO FONOLÓGICO: MEDIDAS DE DURAÇÃO E DESLOCAMENTO DA LÍNGUA
}

\author{
Aline Mara de OLIVEIRA* \\ Larissa Cristina BERTI**
}

- RESUMO: Este estudo caracterizou as medidas de deslocamento e duração da língua no desvio fonológico na produção típica de crianças falantes do português brasileiro. As hipóteses são: (1) crianças com desvio fonológico caracterizam-se por uma duração maior que as crianças típicas; e (2) o deslocamento da língua é maior no desvio fonológico e nas produções típicas das crianças. Participaram cinco crianças com desvio fonológico e cinco com desenvolvimento típico de linguagem. O corpus consistiu em palavras contendo dois padrões silábicos: CCV e CV. As imagens foram capturadas simultaneamente pelo software Articulate Assistant Advanced (AAA). A hipótese 1 não foi confirmada e a 2 foi parcialmente confirmada. A duração é maior nas sílabas CCV. O deslocamento médio e posterior da língua é maior no desvio fonológico. As crianças com desvio fonológico parecem não coordenar os gestos da ponta da língua durante a produção de CCV (esperado para a produção do tepe), entretanto deslocam a região média e a região posterior da língua.

- PALAVRAS-CHAVE: análise articulatória; aquisição de linguagem; aquisição fonológica; português do Brasil.

\section{Introdução}

A ultrassonografia de língua tem se estabelecido como uma técnica nova e útil para analisar a fala, particularmente no que refere à investigação e à intervenção por meio da biofeedback nos distúrbios dos sons da fala (BRESSMANN HENG; IRISH, 2005; CLELAND; SCOBBIE; WRENCH, 2015; BACSFALVI; BERNHARDT, 2011; CLELAND et a.l, 2016). Especialmente, tem-se interesse na análise dos gestos complexos de língua envolvidos na produção de encontros consonantais e nas reduções do encontro consonantal observadas em crianças com desenvolvimento atípico da fala

Universidade Federal de Santa Catarina (UFSC). Centro de Ciências da Saúde. Florianópolis - SC - Brasil. Departamento de Fonoaudiologia. aline.mara.oliveira@ufsc.br. ORCID: 0000-0002-4002-6382.

** Universidade Estadual Paulista (UNESP). Faculdade de Filosofia e Ciências. Marília - SP - Brasil. Departamento de Fonoaudiologia. berti.larissa@gmail.com. ORCID: 0000-0002-4144-2804. 
(ALBANO, 2001; BACSFALVI; BERNHARDT, 2011; ZHARKOVA; GIBBON; LEE, 2017).

A aquisição dos encontros consonantais (CCV) são um desafio para as crianças com desvio fonológico. Pesquisas envolvendo análise acústica de Miranda e Silva (2011) e Mezzomo, Mota e Giacchini (2008) investigaram diferenças entre a produção de encontros consonantais por crianças com desenvolvimento fonológico típico e atípico. Os resultados de Miranda e Silva (2011) demonstraram maior duração na produção das sílabas $\mathrm{CCV}$ realizadas por crianças com desvio fonológico se comparada às crianças com desenvolvimento típico. Esses resultados foram interpretados como um indicativo de que crianças com desvio fonológico fazem uso do alongamento das vogais (alongamento compensatório) para diferenciar entre os dois padrões silábicos: $\mathrm{CCV}$ (percebidos auditivamente como CV pelos ouvintes) e CV. Isso mostra que as crianças com desvio fonológico possuem conhecimento fonológico abstrato sobre as estruturas silábicas.

Mezzomo, Mota e Giacchini (2008) também por meio de pistas acústicas identificaram o uso da estratégia de alongamento compensatório entre crianças com desenvolvimento fonológico típico e atípico durante a produção do encontro consonantal. A análise acústica validou o conhecimento fonológico em relação à presença do encontro consonantal mesmo quando este ainda não está preenchido com os segmentos-alvo (CC), ou seja, a implementação da análise acústica permite a descrever de maneira mais confiável as realizações fonológicas.

Com base em dados de ultrassom e ressonância magnética, Gick et al. (2007) descreveram diferenças articulatórias nas substituições de líquidas em crianças com desvio fonológico. Os autores argumentaram que os erros que envolvem o $[\mathrm{w}]$ para $[\mathrm{r}]$, são o resultado de graus de liberdade linguais reduzidos. Com outro instrumento de análise da articulação, Lee et al. (2014) utilizaram a eletropalatografia para investigar a produção de vogais em crianças com desvio fonológico e típico. Os autores identificaram padrões de contato linguopalatal mais amplos nas crianças com desvio fonológico, indicando pior controle temporal e espacial dos movimentos de língua.

Pesquisas ultrassonográficas anteriores sobre redução de encontros consonantais em crianças com desenvolvimento típico falantes do português brasileiro (VASSOLER; BERTI, 2018; OLIVEIRA; BERTI, 2018) revelaram diferenças nos movimentos da língua entre a produção de encontros consonantais e a redução dos encontros consonantais. Ao comparar a produção dos sons alvo [r] e [1] no padrão silábico CV, por meio da sobreposição dos contornos da língua, Barberena (2016) analisou a produção de crianças com desenvolvimento fonológico típico, atípico e adultos. A autora verificou que durante a produção de [1], os grupos de crianças com desenvolvimento fonológico típico e atípico apresentaram diferença estatística entre as médias dos desvios-padrão dos contornos de língua, o mesmo ocorreu na produção de [r], uma vez que também houve diferença entre os dois grupos para o contexto da vogal/a/. Além disso, observou-se para o grupo de crianças com desvio fonológico, ambos os sons-alvo [r] e [1] apresentaram dois gestos distintos (ponta e raiz da língua), sendo diferentes entre si, pois em [r] 
apresenta maior retração da raiz da língua em direção à faringe e maior elevação da ponta da língua e dorso se comparado à [1]. Como é necessário maior constrição da ponta e da raiz da língua para atingir os resultados acústicos esperados para/f/, o desvio fonológico tende a realizar os sons [1], [j] ou mesmo a omissão do som alvo (/f/), devido à dificuldade de dissociar os gestos articulatórios duplos em um articulador oral único.

Berti, Boer e Bressmann (2016) compararam as produções de crianças com desenvolvimento fonológico típico e atípico, utilizando a medida de deslocamento de língua durante a produção de sons líquidas do português brasileiro (/l/, / / /, / / e /R/ no contexto da vogal /a/). Os resultados mostraram que crianças com desvio fonológico realizaram maior deslocamento de língua na produção de $/ 1 /$ e $/ K /$ se comparado às crianças com desenvolvimento típico. Os autores interpretaram esse resultado como a falta de controle anatômico da língua durante a produção dos segmentos envolvidos (corroborando estudo anterior de Gibbon, 1999).

No que diz respeito à produção das líquidas, no interior do padrão silábico $\mathrm{CCV}$, Vassoler e Berti (2018) analisaram a produção típica e atípica CCV em que o segundo C corresponde a $[\mathrm{r}]$, medindo a área entre a ponta e a lâmina da língua no ponto máximo da constrição do tepe. Os autores identificaram que crianças com desvio fonológico apresentaram, em ambos os padrões silábicos (CCV e CV), maiores valores de área entre ponta e lâmina de língua em comparação com crianças típicas, indicando que crianças com desvio fonológico utilizam maior área da língua, e possivelmente, não diferenciam os gestos articulatórios da ponta e da corpo da língua na produção de CV e CCV.

Em outro estudo, Oliveira e Berti (2018) também investigaram também investigaram a produção de $\mathrm{CV}$ e $\mathrm{CCV}$ de crianças com desenvolvimento fonológico típico e com desvio fonológico por meio de medidas de razões entre a ponta e a lâmina de língua, entre a ponta e o dorso de língua e entre a lâmina e o dorso de língua. O valor positivo da razão entre a ponta e a lâmina da língua significa que a ponta da língua está em uma posição mais elevada em relação à lâmina, enquanto o valor negativo da relação significa que a ponta está mais baixa se comparada à lâmina da língua. Os resultados sugerem que crianças com desenvolvimento típico parecem estar na direção esperada do padrão de sílaba CCV (maior elevação da ponta) quando comparada à sílaba $\mathrm{CV}$. Para as crianças com desvio fonológico, as razões evidenciaram elevação da ponta da língua para ambas as sílabas, sugerindo, para a produção do tepe, a presença de elevação da ponta da língua com magnitude reduzida e com gestos não sobrepostos do alvo CCV (gestos indiferenciados).

Os estudos (VASSOLER; BERTI, 2018; OLIVEIRA; BERTI, 2018) mostraram que maior área e elevação na região anterior da língua de crianças com desvio fonológico se comparam às crianças típicas. Pesquisas envolvendo as consoantes no contexto silábico CV (BERTI; BOER; BRESSMANN, 2016; BARBERENA, 2016; VASSOLER; BERTI, 2018) indicaram que crianças com desvio fonológico apresentam maior deslocamento de língua que pode ser marcado por maior duração.

Assim, é possível verificar que a análise ultrassonográfica quantitativa tem contribuído para diferenciar as produções típicas do desvio fonológico em diferentes sons e 
padrões silábicos. A literatura já aponta que medidas quantitativas ultrassonográficas são capazes de diferenciar grupos de crianças (com desenvolvimento fonológico típico e com desvio fonológico) durante a produção dos sons líquidos tanto no padrão silábico CV (BARBERENA, 2016), quanto no CCV (VASSOLER; BERTI, 2018). Assim, em relação a todas as medidas analisadas anteriormente, pode-se inferir que há uma maior elevação da região anterior da língua durante a produção do CCV nos casos de crianças com desvio fonológico.

No entanto, estudos anteriores não mostraram informações sobre o deslocamento da língua durante a produção desses padrões silábicos. Com base na literatura (VASSOLER; BERTI, 2018; OLIVEIRA; BERTI 2018) de que crianças apresentam maior uso da massa de língua em produções atípicas, resultando em maior deslocamento de língua e em maior duração, esta pesquisa pretende corroborar/refutar achados anteriores, a partir das seguintes hipóteses:

(1) No português brasileiro, as crianças com desvio fonológico são caracterizadas por maiores durações se comparado às crianças típicas;

(2) As crianças com desvio fonológico apresentam maior deslocamento de língua se comparada às produções infantis típicas.

Assim, este estudo teve como objetivo caracterizar o deslocamento de língua nas produções de crianças desvio fonológico e de crianças com desenvolvimento típico falantes do português brasileiro.

\section{Materiais e métodos}

\section{Participantes}

Participaram do estudo 10 participantes falantes do Português Brasileiro (PB): cinco crianças com desenvolvimento fonológico típico e cinco crianças com desvio fonológico (com redução de encontros consonantais). Para ambos os grupos de crianças, os critérios de exclusão foram: ausência de deficiências intelectuais e neurológicos; ausência de alterações anatômicas e morfológicas, que prejudicassem o processo de produção da fala (por exemplo, fenda labial e palatina); e ausência de otologia/distúrbios auditivos.

\section{Procedimentos ou equipamentos}

Os participantes foram gravados, individualmente, em uma única sessão de aproximadamente 30 minutos, no Laboratório de Análises Acústicas (LAAC) da Universidade Estadual Paulista (UNESP - campus de Marília) (São Paulo, Brasil). 
Essas gravações foram realizadas com a utilização de um aparelho de ultrassom portátil (modelo Mindray 6600) localizado em uma sala acusticamente tratada. As imagens de ultrassom foram adquiridas com uma frequência de imagem de 6,5 MHz e um ângulo de $120^{\circ}$. O aparelho de ultrassom produziu uma alimentação de vídeo NTSC com uma taxa de frames de $29.97 \mathrm{~Hz}$. O transdutor de ultrassom foi estabilizado por meio do estabilizador de cabeça (Articulate Instruments, Edinburgh, UK). O áudio foi gravado através de um microfone dinâmico Shure 8800 (Shure Inc., Niles, IL, EUA).

As imagens de ultrassom e o sinal sonoro foram gravados diretamente no disco rígido de um computador pessoal. O software Articulate Assistant Advanced (AAA) (Articulate Instruments, Edinburgh, UK) foi utilizado para capturar as imagens de ultrassom e o áudio. O software também sincronizou as gravações de vídeo e áudio.

\section{Estímulos}

Este estudo teve como objetivo analisar a produção de tepe no interior dos encontros consonantais $(\mathrm{CrV})$ versus $(\mathrm{CV})$. As crianças gravaram um corpus composto por nove pares de palavras contendo o padrão silábico CCV e CV: broa ['broe]; boa ['bov]; prato

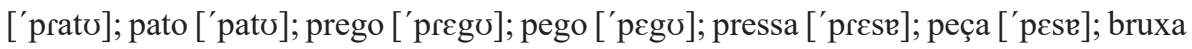
['brufe]; bucha ['bufe]; troco ['trokv]; toco ['tokv]; grato ['grato]; gato ['gato]; frita ['frite]; fita ['fite]. O critério de seleção das palavras foi escolhido a partir do padrão silábico $\mathrm{CCV}$ e $\mathrm{CV}$, obedecendo aos critérios de alta frequência lexical no português brasileiro (MIRANDA; SILVA, 2011).

As figuras referentes aos pares mínimos foram apresentadas aos indivíduos por meio de uma atividade lúdica, ou seja, a criança visualizava a imagem e na tela $A A A$ e pronunciava a palavra-alvo correspondente. Antes do início da gravação, a identificação de algumas palavras-alvo como "grato, pego e boa" (verbo e adjetivo) foram mencionadas pelo avaliador. Se a criança produzisse uma palavra divergente da palavra esperada, o pesquisador solicitava uma imitação da palavra alvo.

Para cada palavra, as crianças foram solicitadas em produzir cinco repetições, sendo utilizadas as três melhores gravações no que refere à qualidade da imagem. $\mathrm{O}$ número total de estímulos registrados foi: 540 repetições (18 palavras x 3 repetições x 5 crianças com desvio fonológico +5 crianças desenvolvimento fonológico típico).

\section{Análise perceptivo-auditiva}

As durações das sílabas foram exportadas do programa Articulate Assistant Advanced. Três fonoaudiólogos com experiência em transcrição fonética realizam o julgamento perceptivo-auditivo (juízes residentes na mesma região dialetal dos participantes). Os 540 estímulos foram organizados de modo randomizado na tentativa de evitar padrões de resposta no julgamento. O juiz tinha três opções de resposta entre $\mathrm{CCV}, \mathrm{CV}$ ou outra opção (neste caso seria necessário transcrever). 


\section{Medidas de duração}

Os arquivos de som foram editados e codificados no software $A A A$, em seguida, foram exportados e analisados pelo software PRAAT (BOERSMA; WEENINK, 2014). Os parâmetros acústicos utilizados foram: a duração absoluta das sílabas CCV e CV e a duração relativa dos pares silábicos. A segmentação foi baseada na duração da consoante plosiva (no burst) ou da fricativa (no ruído da fricativa) até o final da vogal subsequente.

\section{Medidas de ultrassom}

Um total de 540 (10 participantes $\times 3$ repetições $\times 18$ palavras $)$ imagens de ultrassom foram segmentadas por meio do software AAA. Os frames dos vídeos ultrassonográficos foram identificados e o contorno de da língua foi traçado de modo semi automático usando o $A A A$.

$\mathrm{Na}$ sílaba $\mathrm{CCV}$, identificou-se o frame correspondente ao ponto máximo da língua do referente à realização do tepe e o frame da vogal seguinte. Da mesma forma, na sílaba $\mathrm{CV}$, identificou-se o frame relacionado ao ponto máximo de constrição da língua entre a consoante e a vogal (onde estaria o tepe) e o frame do centro do vogal seguinte.

A altura da língua (em milímetros) foi feita ao longo de 42 linhas radiais ${ }^{1}$ espaçadas igualmente gerando 120 graus. O limiar de confiança escolhido foi de $90 \%$, sendo que os valores abaixo de $90 \%$ foram excluídos devido à má qualidade do contorno da língua.

A análise foi baseada em um estudo prévio (BERTI; BOER; BRESSMANN, 2016). Os dados das 28 linhas do leque central (entre a 8 e a 35 linhas do leque) foram incluídos na análise, uma vez que as linhas mais anteriores (abaixo da oitava) ou as mais posteriores (acima de 35) não continham medidas de altura da língua confiáveis. Os autores desenvolveram uma medida global de deslocamento da língua para comparar o contorno de língua de diferentes falantes. A medida de deslocamento foi calculada dividindo os valores da altura da língua ao longo das linhas que compõem o leque do tepe na sílaba CCV (ou entre a consoante e a vogal na sílaba CV) pelos valores da altura da língua para a vogal seguinte, resultando em uma porcentagem de deslocamento da língua.

O deslocamento foi calculado pela média das diferenças percentuais absolutas entre a linha 8 e 35 do leque que compõem o frame. Todas as células que continham divisões por 0 foram removidas. Da mesma forma, também foram calculadas a porcentagem de deslocamento da língua da metade anterior (8 e 17 linhas) e da metade posterior (17 e 35 linhas de grade) do intervalo selecionado.

O software $A A A$ divide a imagem de ultrassom em 42 linhas paralelas a partir do ângulo de $90^{\circ}$ dispostas em posições inclinadas semelhantes a um leque. 


\section{Análise estatística}

A análise inferencial foi realizada por meio do programa Statistical Package for the Social Sciences (SPSS, IBM). As medidas acústicas e articulatórias foram analisadas com a ANOVA com medidas repetidas. A variável inter sujeito foi o padrão silábico (CV e CCV) e a variável intra sujeito foi a condição clínica (desenvolvimento fonológico típico versus desenvolvimento fonológico atípico).

Para as ANOVAs de medidas repetidas, os valores de $\mathrm{p}$ foram considerados significativos se fossem menores que 0,05 . Como teste post hoc, foi aplicado o teste de Bonferroni.

\section{Resultados}

\section{Medidas}

\section{Análise perceptivo-auditiva}

Das 270 palavras composta por CCV, 135 (100\%) realizadas por crianças com desenvolvimento fonológico típico foram avaliadas na análise perceptivo-auditiva $\mathrm{CCV}$, enquanto $135(100 \%)$ das produções de crianças com desvio fonológico foram avaliadas como $\mathrm{CV}$.

Das 270 palavras com início de CV, 135 (100\%) das produções realizadas por crianças com desenvolvimento fonológico típico foram julgadas como $\mathrm{CV}$ e as 135 (100\%) produções das crianças com desenvolvimento fonológico atípico foram avaliadas como $\mathrm{CV}$.

\section{Duração absoluta}

Os resumos dos resultados da duração absoluta das sílabas CCV e CV estão ilustrados na Tabela 1:

Tabela 1 - Caracterização das crianças que participaram da investigação.

\begin{tabular}{|c|c|c|c|}
\hline Condição Clínica & Crianças & Gênero & Idade \\
\hline Desenvolvimento fonológico típico & 1 & Feminino & 5 anos e 4 meses \\
\hline Desenvolvimento fonológico típico & 2 & Feminino & 6 anos e 8 meses \\
\hline Desenvolvimento fonológico típico & 3 & Feminino & 5 anos e 6 meses \\
\hline Desenvolvimento fonológico típico & 4 & Masculino & 6 anos e 3 meses \\
\hline Desenvolvimento fonológico típico & 5 & Feminino & 6 anos e 2 meses \\
\hline
\end{tabular}




\begin{tabular}{|c|c|c|c|}
\hline Condição Clínica & Crianças & Gênero & Idade \\
\hline Desvio Fonológico & 6 & Feminino & 6 anos \\
\hline Desvio Fonológico & 7 & Masculino & 6 anos e 9 meses \\
\hline Desvio Fonológico & 8 & Masculino & 6 anos e 7 meses \\
\hline Desvio Fonológico & 9 & Masculino & 5 anos e 5 meses \\
\hline Desvio Fonológico & 10 & Feminino & 5 anos e 5 meses \\
\hline
\end{tabular}

Fonte: Elaboração própria.

A ANOVA de medidas repetidas foi calculada para duração absoluta. Um efeito significativo entre os sujeito foi encontrado para o padrão de sílaba $(\mathrm{F}=40.6, \mathrm{df}=1.87$, $\mathrm{p}<0.00$ ) e não houve efeito significativo para o grupo (desenvolvimento fonológico típico versus desenvolvimento fonológico atípico) $(\mathrm{F}=3.81, \mathrm{df}=1.87, \mathrm{p}=0.05)$.

A interação entre o grupo e o padrão de sílaba abordado teve significância estatística $(\mathrm{F}=12,50, \mathrm{df}=1,87, \mathrm{p}<0,00)$. O teste post-hoc Bonferroni demonstrou que as sílabas CCV são significativamente maiores do que as sílabas $\mathrm{CV}(\mathrm{p}<0,00)$ na produção de crianças com desenvolvimento típico. A Figura 1 mostra a duração das sílabas CCV e CV nos dois grupos de crianças:

Figura 1 - Duração do CCV e CV alvo por grupos.

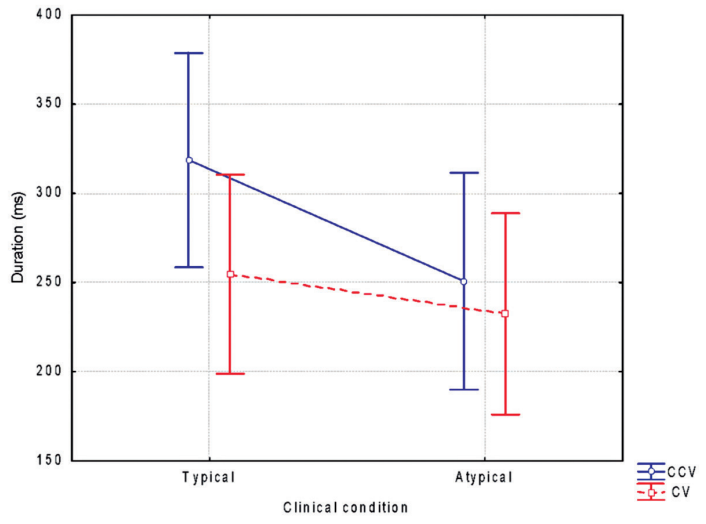

Fonte: Elaboração própria.

\section{Deslocamento de língua}

Os resumos dos resultados para médias e desvios-padrão para a média, deslocamentos anterior e posterior são mostrados na Tabela 2: 
Tabela 2 - Duração da sílaba alvo do CCV e da sílaba CV de crianças típicas e atípicas.

\begin{tabular}{|c|c|c|}
\hline \multirow{2}{*}{ Condição Clínica } & \multicolumn{2}{|c|}{ Duração absoluta } \\
\cline { 2 - 3 } & CCV & CV \\
\hline $\begin{array}{c}\text { Desenvolvimento } \\
\text { fonológico típico }\end{array}$ & $318.62(118.63)$ & $254.55(111.79)$ \\
\hline Desvio Fonológico & $249.43(124.74)$ & $231.14(112.91)$ \\
\hline
\end{tabular}

Fonte: Elaboração própria.

Para o deslocamento médio, ANOVA de medidas repetidas demonstrou um efeito intraindividual significativo para a condição clínica $(\mathrm{F}=17,37, \mathrm{df}=1,57, \mathrm{p}<0,00)$ e o teste não mostra efeito intra sujeito para o padrão de sílaba $(\mathrm{F}=65,14, \mathrm{df}=1,57, \mathrm{p}=1,00)$. A interação entre o grupo clínico e o padrão silábico não foi estatisticamente significativa $(\mathrm{F}=12,21, \mathrm{df}=1,5, \mathrm{p}=1,00)$. Os testes post-hoc demonstraram que o deslocamento de língua das crianças com desvio fonológico foi significativamente maior se comparado às crianças com desenvolvimento fonológico típico $(\mathrm{p}<0,00)$.

A Figura 2 mostra o deslocamento médio de língua dos padrões silábicos CCV e CV:

Figura 2 - Box-plot referente ao deslocamento médio de língua durante a produção de CCV e CV.



Fonte: Elaboração própria.

Para o deslocamento anterior de língua, a ANOVA de medidas repetidas não mostrou efeito inter sujeito para o padrão silábico $(\mathrm{F}=0,63, \mathrm{df}=1,6, \mathrm{p}=0,43)$ e não houve diferenças entre sujeitos para os grupos de crianças $(\mathrm{F}=0,64, \mathrm{df}=1,58, \mathrm{p}=0,42)$. A interação entre grupo clínico e padrão silábico não teve significância estatística $(\mathrm{F}=1,31, \mathrm{df}=1,58, \mathrm{p}=0,25)$. A Figura 3 mostra o deslocamento da região anterior da língua durante as sílabas CCV e CV realizados pelos grupos de crianças: 
Figura 3 - Box-plot referente ao deslocamento anterior de língua durante a produção de $\mathrm{CCV}$ e $\mathrm{CV}$.



Fonte: Elaboração própria.

No que se refere ao deslocamento da região posterior de língua, a ANOVA por medidas repetidas demonstrou significância estatística de condição clínica entre sujeitos (desvio fonológico e crianças desenvolvimento fonológico atípico) ( $\mathrm{F}=6,00, \mathrm{df}=1,62$, $\mathrm{p}=0,01)$, mas no efeito intra sujeito não foi identificado significância estatística para o padrão silábico $(\mathrm{F}=0,32, \mathrm{df}=1,62, \mathrm{p}=0,56)$. A interação entre o grupo e o padrão silábico não relevou significância estatística $(\mathrm{F}=3,58, \mathrm{df}=1,62, \mathrm{p}=0,06)$.

A Figura 4 ilustra o deslocamento da região posterior da língua das sílabas CCV e CV:

Figura 4 - Box-plot referente ao deslocamento posterior de língua durante a produção de $\mathrm{CCV}$ e $\mathrm{CV}$.

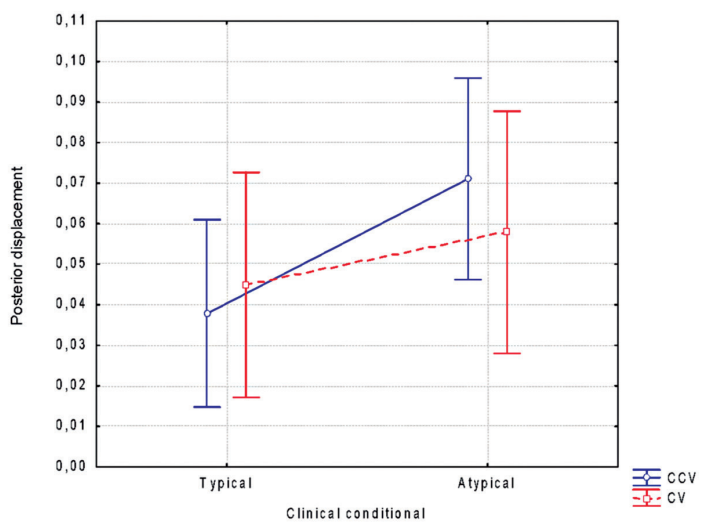

Fonte: Elaboração própria. 


\section{Discussão}

O objetivo do presente estudo foi caracterizar a produção de crianças com desenvolvimento fonológico típico e atípico no português brasileiro. As hipóteses de pesquisa permearam que a duração das sílabas com crianças com desvio fonológico são caracterizadas maiores se comparado ao desenvolvimento fonológico típico (primeira hipótese) e o deslocamento de língua é maior no desvio fonológico do que nas produções infantis típicas (segunda hipótese).

De acordo com a primeira hipótese, as produções das crianças com desenvolvimento fonológico típico e atípica. A hipótese não foi confirmada.

As medidas de duração demonstraram que as sílabas CCV eram significativamente mais longas do que as sílabas CV no desenvolvimento típico, confirmando achados anteriores (EDWARDS et al., 1999). Em contrapartida, outros estudos (MEZZOMO; MOTA; GIACCHINI, 2008; MIRANDA; SILVA 2011; GIACCHINI; MOTA; MEZZOMO, 2011) identificaram maior duração nas produções de crianças com desvio fonológico. Embora não tenha havido diferença estatística entre os participantes do estudo, esses grupos de crianças parecem ter iniciado a diferenciação do padrão CCV e $\mathrm{CV}$, uma vez que numericamente as sílabas CCV são mais longas que as sílabas CV. No entanto, essa diferença não foi robusta o suficiente para ser marcada estatisticamente, conforme demonstrado por Oliveira e Berti (2018).

A segunda hipótese de que o deslocamento de língua é maior no desvio fonológico e nas produções típicas de crianças foi confirmada parcialmente.

Os valores de deslocamento médio da língua e da região posterior da língua foram diferentes entre os grupos (desenvolvimento fonológico típico versus desenvolvimento fonológico atípico). As crianças com desvio fonológico apresentaram maior deslocamento de língua se comparado às produções das crianças típicas. Porém, para as medidas de deslocamento anterior da língua não houve diferença entre os grupos, ou seja, as produções das crianças típicas e atípicas apresentaram similaridade no deslocamento da região anterior da língua.

Embora as crianças apresentem estruturas anatômicas semelhantes, independentemente da condição clínica, foi possível encontrar diferenças na porcentagem do deslocamento média de /r/ entre os dois grupos de crianças. Uma possível explicação para a diferença na produção de crianças típicas e atípicas pode estar associada à presença de gestos linguais indiferenciados (GIBBON, 1999; VASSOLER; BERTI 2018; OLIVEIRA; BERTI 2018), durante a produção de fala das crianças desenvolvimento fonológico atípico. Os gestos linguais indiferenciados se manifestam quando a língua entra em contato simultaneamente com a região anterior e posterior do palato, ou quando toda a superfície da língua entra em contato com o palato, causando uma falta de distinção entre a ponta da língua e o dorso e as margens laterais da língua contra o palato (GIBBON, 1999; VASSOLER; BERTI, 2018), ou seja, as crianças têm dificuldade em distinguir entre os gestos coronal e dorsal da língua, indicando novamente um problema com o controle motor preciso necessário para a fala inteligível. 
Gibbon (1999) realizou um extenso artigo de revisão da literatura sobre gestos indiferenciados que envolviam a análise por meio da eletropalatografia (EPG). Além de definir e caracterizar a terminologia de gestos linguais indiferenciados (GGs) (conforme citado no parágrafo anterior), a autora identificou na literatura relata que em relação a 17 crianças com desvio fonológico (ou articulatório), 12 crianças com gestos linguais indiferenciados, não percebidos análise de oitiva (transcrições fonéticas), porém foram detectados pelo EPG. O estudo indicou que a presença de GGs reflete dificuldades no nível motor (fonético) devido ao controle imaturo da língua.

Outro estudo anterior (MCALLISTER, 2012) também envolvendo ultrassonografia de língua, identificou que as crianças são conhecidas por terem dificuldade em executar movimentos discretos da língua, preferindo movimentar a língua e a mandíbula como uma única unidade. Isso predispõe a criança a reduzir o contato linguopalatal indiferenciado, neutralizando o contraste entre o som coronal e o som velar (MCALLISTER, 2012).

Em relação à produção da fala de crianças com desenvolvimento fonológico esperado, a ponta da língua e o corpo da língua são articuladores quase independentes, podendo produzir padrões bem definidos contra o palato. Porém, quando as crianças apresentam os gestos linguais indiferenciados, elas são incapazes de diferenciar os gestos da ponta e da região anterior do corpo da língua. Os gestos linguais indiferenciados podem ser entendidos como reflexo de restrições motoras na fala, em decorrência de atrasos ou desvios no controle dessas regiões da língua (GIBBON, 1999).

Em um estudo envolvendo eletropalatografia, Gouzee et al. (2007) identificou em crianças com gestos linguais indiferenciados, corpo excessivo do movimento da língua durante a produção da consoante /t/. Os autores acreditam que o corpo excessivo da língua durante o movimento pode ser decorrente do resultado de um controle motor pobre, imaturo ou desviante do sistema de alavanca e/ou pode ser devido ao mecanismo de compensação para neutralizar as dificuldades do controle motor fino de ponta da língua.

Os resultados deste estudo corroboram com Goozée et al. (2007), que mostram que crianças com desvio fonológico não parecem coordenar gestos de ponta da língua e corpo durante a produção CCV, o que pode ser interpretado o uso excessivo de língua devido aos gestos linguais indiferenciados.

\section{Conclusão}

Este estudo caracterizou o deslocamento de língua nas produções de crianças com desvio fonológico e de crianças com desenvolvimento típico falantes do português brasileiro. As medidas ultrassonográficas envolveram as medidas de deslocamento médio, de deslocamento anterior e de deslocamento posterior da língua durante a produção das sílabas $\mathrm{CV}$ e CCV por crianças com desvio fonológico e crianças com desenvolvimento típico.

As durações das sílabas CCV são significativamente maiores se comparadas às sílabas CV e ao deslocamento da língua entre as produções infantis típicas e atípicas. 
O deslocamento médio da língua é maior no desvio fonológico. Além disso, crianças com desvio fonológico parecem não coordenar os gestos de ponta e de dorso da língua separadamente durante a produção do $\mathrm{CCV}$, o que pode ser interpretado como maior uso da língua devido aos gestos linguais indiferenciados.

Como pode ser observado, a ultrassonografia é uma ferramenta útil para avaliar e caracterizar a produção de fala típica e atípica. A investigação dos movimentos da língua durante a fala é altamente recomendável para observar os erros da fala, fornecendo informações para a visualização direta dos articuladores. É uma técnica não invasiva, segura, rápida e de baixo custo.

\section{Agradecimentos}

Esta pesquisa foi financiada pela FAPESP - Agência de Pesquisa do Estado de São Paulo (bolsas no 13 / 00513-8 e 14 / 09605-5). Esta pesquisa foi possível graças às crianças e seus pais que generosamente aceitaram participar do estudo, bem como aos auxiliares de pesquisa, fonoaudiólogos e alunos de fonoaudiologia que fizeram parte do projeto. Os autores gostariam de agradecer o financiamento da FAPESP, da Agência de Pesquisa do Estado de São Paulo (bolsa no 13 / 00513-8 e 14 / 09605-5) e do Professor Dr. Tim Bressmann (Universidade de Toronto) pela contribuição na análise dos dados desta pesquisa.

OLIVEIRA, A.; BERTI, L. Tongue movement in consonant cluster reduction in brazilian children with phonological disorder: duration and tongue displacement measures. Alfa, São Paulo, v.66, 2022.

- ABSTRACT: This study characterized tongue displacement and duration measures in phonological disorder and typical children's productions of Brazilian Portuguese speakers. The hypotheses are: (1) children with phonological disorder are characterized by longer duration than typical children; and (2) tongue displacement is greater in phonological disorder and typical children's productions. Participants were five children diagnosed with phonological disorders (PD) and five typically developed (TD). The corpus consisted of three repetitions with two syllable patterns: $C C V$ and $C V$. The images were captured simultaneously by the Articulate Assistant Advanced software. The hypothesis 1 was not confirmed and hypothesis 2 was partially confirmed. The durational measures demonstrated that the CCV syllables were significantly longer than the CV syllables in TD. The posterior and average tongue displacement is greater in phonological disorder. The children with phonological disorders do not seem to coordinate gestures of the tongue tip and dorsum during CCV production separately (expected for the production of tap), however they displaced the average and posterior region of the tongue.

- KEYWORDS: articulatory analysis; language acquisition; phonological acquisition; brazilian portuguese. 


\section{REFERÊNCIAS}

ALBANO, E. C. O gesto e suas bordas: esboço de fonologia acústico-articulatória do português brasileiro. Campinas: Mercado das Letras, 2001.

ARTICULATE INSTRUMENTS LTD. Articulate assistant advanced user guide: Version 2.14. Edinburgh: Articulate Instruments, 2014.

ARTICULATE INSTRUMENTS LTD. Ultrasound stabilisation headset users manual: 1.4. Edinburgh: Articulate Instruments, 2008.

BACSFALVI, P.; BERNHARDT, B. M. Long-term outcomes of speech therapy for seven adolescents with visual feedback technologies: Ultrasound and electropalatography. Clinical Linguistics and Phonetics, London, v.25, p.1034-1043, 2011.

BARBERENA, L. S. Caracterização de aspectos da produção articulatória do [[] tap por análise instrumental e resultados de intervenção. Tese (Doutorado em Distúrbios da Comunicação Humana) - Universidade Federal de Santa Maria, Santa Maria, 2016.

BRESSMANN, T.; HENG, C.; IRISH, J. C. Applications of 2D and 3D ultrasound imaging in speech-language pathology. Journal of Speech Language Pathology and Audiology, [s.l.], v.29, p.158-168, 2005.

BERTI, L.; BOER, G. D.; BRESSMANN, T. Tongue displacement and durational characteristics of normal and disordered Brazilian Portuguese liquids. Clinical Linguistics and Phonetics, London, v.30, p.131-49, 2016.

BOERSMA, P.; WEENINK, D. PRAAT: doing phonetics by computer [computer program]. Amsterdam: University of Amsterdam, 2016. Disponível: http://www.praat. org/. Acesso em: 04 nov. 2021.

CLELAND, J.; SCOBBIE, J. M.; WRENCH, A. A. Using ultrasound visual biofeedback to treat persistent primary speech sound disorders. Clinical Linguistics \& Phonetics, London, v.29, p.575-597, 2015.

CLELAND, J. et al. Covert contrast and covert errors in persistent velar fronting. Clinical Linguistics \& Phonetics, London, v.31, p.1-21, 2016.

EDWARDS, J. et al. Characterizing knowledge deficits in phonological disorders. Journal of Speech, Language, and Hearing Research, Rockville, v.42, p.169-86, 1999.

GIACCHINI, V.; MOTA, H. B.; MEZZOMO, C. L. Diferentes modelos de terapia fonoaudiológica nos casos de simplificação do onset complexo com alongamento compensatório. Revista Cefac, São Paulo, v.13, p.57-64, 2011. 
GIBBON, F. E. Undifferentiated lingual gestures in children with articulation/ phonological disorders. Journal of Speech, Language, and Hearing Research, Rockville, v.42, p.382-397, 1999.

GICK, B. et al. A motor differentiation model for liquid substitutions in in children's speech. Proceedings of Meetings on Acoustics, Melville, v.1, p.1-9, 2007.

GOOZÉE, J. et al. Lingual kinematics and coordination in speech disordered children exhibiting differentiated versus undifferentiated lingual gestures. International Journal of Language \& Communication Disorders, London, v.42, p.703-24, 2007.

LEE, A. et al. Tongue-palate contact during selected vowels in children with speech sound disorders. International Journal of Speech-Language Pathology, Abingdon, v.16, p.562-70, 2014.

MCALLISTER, B. T. Positional velar fronting: an updated articulatory account. Journal of Child Language, Cambridge, v.39, p.1043-1076, 2012.

MEZZOMO, C.; MOTA, R. D.; GIACCHINI, V. O uso da estratégia de alongamento compensatório em crianças com desenvolvimento fonológico normal e desviante. Letras de Hoje, Porto Alegre, v.43, p.35-41, 2008.

MIRANDA, I. C. C.; SILVA, T. C. Aquisição de encontros consonantais tautossilábicos: uma abordagem multirrepresentacional. Revista Lingüística, Rio de Janeiro, v.7, p.1-17, 2011.

OLIVEIRA, A. M. O; BERTI, L. C. Aquisição fonológica típica e atípica do padrão silábico CCV: dados acústicos e articulatórios. Alfa, São Paulo, v.62, p.591-612, 2018.

VASSOLER, A. M. O.; BERTI, L. C. Padrões silábicos no desenvolvimento fonológico típico e atípico: análise ultrassonográfica. CoDAS, São Paulo, v.30, p.1-7, 2018.

ZHARKOVA, N.; GIBBON, F. E.; LEE, A. Using ultrasound tongue imaging to identify covert contrasts in children's speech. Clinical Linguistics \& Phonetics, London, v.31, p.21-34, 2017.

Recebido em 7 de fevereiro de 2020

Aprovado em 29 de agosto de 2020 